\title{
Does Extrinsic Goal Framing Enhance Extrinsic Goal-Oriented Individuals' Learning and Performance? An Experimental Test of the Match Perspective Versus Self-Determination Theory
}

\author{
Maarten Vansteenkiste \\ Ghent University \\ Bart Soenens \\ Ghent University
}

\author{
Tinneke Timmermans and Willy Lens \\ University of Leuven
}

Anja Van den Broeck
University of Leuven

\begin{abstract}
Previous work within self-determination theory has shown that experimentally framing a learning activity in terms of extrinsic rather than intrinsic goals results in poorer conceptual learning and performance, presumably because extrinsic goal framing detracts attention from the learning activity and is less directly satisfying of basic psychological needs. According to the match perspective, experimental extrinsic, compared to intrinsic, goal framing should enhance learning and performance for learners who personally hold a stronger extrinsic than intrinsic goal orientation, as these learners' personally held goals match with the situationally induced goals. An experimental field study among 5th-6th grade children shows that extrinsic goal framing resulted in poorer autonomous motivation, conceptual (but not rote) learning, and persistence compared to intrinsic goal framing, irrespective of participants' personal intrinsic versus extrinsic goal orientations and their spontaneous perception of the learning activity as serving an intrinsic or an extrinsic goal. The authors conclude that teachers can best promote intrinsic goals, even when facing students who personally hold a stronger extrinsic than intrinsic goal orientation.
\end{abstract}

Keywords: intrinsic versus extrinsic goals, match perspective, self-determination theory

Values represent categories of desirable life goals, varying in importance, that serve as a guiding principle in people's lives (De Witte, 2004; Rohan, 2000; Rokeach, 1973; Schwartz, 1992, 1999). Various researchers have developed multidimensional models of the interpersonal variation in the type of values (Schwartz, 1994) or life goals (Grouzet et al., 2006; Nuttin \& Lens, 1985) that people hold. ${ }^{1}$ In addition, researchers have been interested in studying the implications of people's life goals for their well-being and achievement. In doing so, they have proposed at least two different and conflicting perspectives. Some researchers, drawing on SDT (Deci \& Ryan, 1985, 2000; Ryan \& Deci, 2000b), hold that some goals are better than others for people's thriving and optimal functioning (Kasser, 2002; Vansteenkiste, Lens, \& Deci, 2006). Other researchers claim that there does not exist a category of more adaptive values or life goals (e.g., Bianco, Higgins, \& Klem, 2003; Sagiv \& Schwartz, 2000; Walsh \& Holland, 1992). They have argued that the relation between people's life goals,

Maarten Vansteenkiste and Bart Soenens, Department of Psychology, Ghent University, Ghent, Belgium; Tinneke Timmermans, Willy Lens, and Anja Van den Broeck, Department of Psychology, University of Leuven, Leuven, Belgium.

Anja Van den Broeck's contributions were supported by the Grant for Scientific Research Flanders.

Correspondence concerning this article should be addressed to Maarten Vansteenkiste, H. Dunantlaan 2, 9000, Ghent, Belgium. E-mail: maarten.vansteenkiste@ugent.be achievement, and adjustment depends on the match or fit between people's personal goal orientations and the goals that are emphasized and encouraged in the direct environment (Pervin, 1968; Schneider, 1987). This viewpoint is commonly referred to as the match perspective.

A few previous studies have directly examined the conflicting hypotheses that can be derived from SDT and the match perspective (Kasser \& Ahuvia, 2002; Sagiv \& Schwartz, 2000; Vansteenkiste, Duriez, Simons, \& Soenens, 2006), but they were all correlational in nature and focused on well-being outcomes only. The current contribution goes beyond past work by providing evidence from an experimental field study that focused on learning and performance outcomes. Before detailing the aims of the present research, we introduce both theoretical perspectives.

\footnotetext{
${ }^{1}$ It should be noted that there exists a slight conceptual difference between values and life goals, as the latter are more concrete and lower order units, whereas the former are rather abstract and higher order units (Grouzet et al., 2006; Schwartz, 1994). The conceptual difference between the constructs is, however, of less importance to the present study, which focuses on the match between personally held life goals or values and the type of life goals or values that are promoted by the social environment. We adopted the term goals throughout the article because this is the term that is most commonly used within research on self-determination theory (SDT)
} 


\section{Self-Determination Theory}

\section{Intrinsic and Extrinsic Motivation and Intrinsic and Extrinsic Goal Contents}

The classic motivation constructs that have received the most empirical and theoretical attention within SDT and other motivational frameworks are intrinsic and extrinsic motivation (Deci \& Ryan, 2000; Vansteenkiste, Lens, \& Deci, 2006). Whereas intrinsic motivation refers to engaging in an activity for its own sake, simply because it is enjoyable and gratifying by itself, extrinsic motivation refers to engaging in an activity to obtain an outcome separable from the activity itself (Ryan \& Deci, 2000a). In earlier theorizing in the 1970s (e.g., Lepper \& Greene, 1978), intrinsic and extrinsic motivation were considered as antipodes, with intrinsic motivation representing the most autonomous or selfdetermined type of motivation and extrinsic motivation representing a nonautonomous or controlled type of motivation. However, subsequent empirical work in the 1980s (e.g., Ryan, Mims, \& Koestner, 1983) made clear that not all extrinsically motivated behavior is nonautonomous, given that individuals are able to volitionally endorse (i.e., internalize) the reasons for engaging in the activity at hand. This insight resulted in a conceptual shift within SDT away from the intrinsic-extrinsic motivation dichotomy toward the distinction between autonomous motivation, which involves both intrinsic and well-internalized motivation, and controlled motivation, which involves poorly internalized or noninternalized motivation. Thus, autonomous and controlled motivation represent two qualitatively different types of reasons for regulating one's behavior and are often studied under the label of the "why" of behavior (Deci \& Ryan, 2000; Vansteenkiste, Lens, \& Deci, 2006; Vansteenkiste, Ryan, \& Deci, in press).

Parallel to the growing attention to the concepts of autonomous and controlled motivation, SDT researchers have also been paying attention to people's goal contents or goal orientations (Kasser \& Ryan, 1993). In this respect, Kasser and Ryan (1993, 1996) differentiated extrinsic goals, such as wealth, physical appeal, and social recognition, from intrinsic goals, such as affiliation, personal growth, and community contribution, on the basis of humanistic (Maslow, 1954) and existential (Fromm, 1976) theorizing. Intrinsic goals reflect people's natural growth tendencies and are characterized by an inwardly oriented frame. In contrast, when people pursue extrinsic goals, they tend to adopt an outward orientation (Williams, Cox, Hedberg, \& Deci, 2000)—that is, they are focused on impressing others by garnering external signs of self-worth. These different goal contents are often studied under the label of the "what" of behavior within SDT (Deci \& Ryan, 2000).

According to SDT, intrinsic and extrinsic goals, which represent qualitatively different types of goals, can be motivated by autonomous or controlled reasons, which represent qualitatively different types of motivations and thus distinct sets of constructs. For instance, a person can be altruistic (i.e., an intrinsic goal) because he or she enjoys pursuing this goal (autonomous motivation) or because he or she would feel bad if he or she did not help people in need (controlled motivation). Similarly, a person can be focused on becoming famous (i.e., an extrinsic goal) because his or her parents pressure him or her to be a well-known person (controlled motivation) or because he or she personally values fame (autonomous motivation).
In the present research, we explicitly focused on the concepts of intrinsic and extrinsic goal orientations. More specifically, we examined whether framing a learning activity in terms of the attainment of intrinsic or extrinsic goals would yield a differential impact on the learning and persistence of intrinsic and extrinsic goal-oriented individuals. To clarify this overall aim, we first elaborate more on the concepts of intrinsic and extrinsic goal contents and their effects, as they have been studied from the SDT perspective.

\section{The Effects of Intrinsic Versus Extrinsic Goal Contents}

SDT starts with the central assumption that all individuals are born with the basic psychological needs for autonomy, competence, and relatedness. The satisfaction of these psychological needs is regarded as necessary nutriments for individuals' optimal performance, well-being, and development, much as plants need water and sunshine to flower (Deci \& Ryan, 2000; Ryan \& Deci, 2000b). To understand the effects of goal contents on people's optimal functioning, one needs to answer the question of how goal pursuit is related to the satisfaction of the basic psychological needs. In this respect, intrinsic goals are said to facilitate psychological well-being, because they provide direct satisfaction of the basic and universal psychological needs for competence, autonomy, and relatedness (Deci \& Ryan, 2000; Kasser, 2002). For instance, when people endorse the intrinsic goal of selfdevelopment, they are more likely to engage in challenging activities in a task-focused manner, which increases their chances of gaining a sense of competence. When people value the development of close relationships, they are more likely to empathically take the perspective of others, which might facilitate a sense of mutual connectedness and thrust (Kasser, 2002; Vansteenkiste, Neyrinck, et al., 2007; Vansteenkiste, Soenens, \& Lens, 2007).

Although extrinsic goal pursuit might provide some satisfaction, this type of satisfaction is likely to be derivative and short lived, because extrinsic goal pursuits do not directly satisfy individuals' basic psychological needs (Deci \& Ryan, 2000; Kasser, 2002; Kasser, Ryan, Couchman, \& Sheldon, 2004). For instance, extrinsic goal-oriented individuals tend to approach others in an "objectifying" manner (Kasser, 2002) — that is, to treat them as objects that should be used in the most efficient way toward one's extrinsic goal attainment. Further, (learning) activities are approached in a restricted and rigid manner (Vansteenkiste, Simons, Lens, Soenens, \& Matos, 2005); that is, extrinsic goal-oriented individuals only put effort into the activity as far as this will help them in achieving their extrinsic ambitions. Thus, they are less deeply involved in learning tasks because engagement in such tasks is only valued to the extent that they are instrumental for reaching extrinsic goals (Vansteenkiste, Simons, Lens, Soenens, et al., 2004). Such an objectifying and narrow-focused approach is, however, unlikely to create opportunities for satisfaction of the basic psychological needs for relatedness, competence, and autonomy. Consistent with these claims, Vansteenkiste, Neyrinck, et al. (2007) found in a sample of employees that an extrinsic, relative to an intrinsic, goal pursuit hindered basic need satisfaction.

Because of their differential linkage to the satisfaction of basic psychological needs, intrinsic versus extrinsic goals differentially predict psychological well-being (Ryan, Sheldon, Kasser, \& Deci, 1996). In line with this reasoning, previous research has docu- 
mented that when participants' life aspirations are more strongly centered around the pursuit of extrinsic rather than intrinsic goals, they display lower psychological well-being and social adjustment and are more vulnerable to ill-being and drug and alcohol abuse (see Kasser, 2002; Kasser et al., 2004, for an overview). Although most of this work has been conducted among late adolescent and adult samples, a few studies have suggested that early adolescents (Cohen \& Cohen, 1996) and children (Kasser, 2005) also suffer emotionally from adopting an extrinsic or materialistic goal orientation.

It is interesting that SDT maintains that the pursuit of extrinsic, relative to intrinsic, goals should hinder the learning process and individuals' broader psychosocial adjustment, even when extrinsic goals are highly emphasized by the social environment - that is, in spite of a person-environment match in extrinsic goals. This is because extrinsic goals, by their very nature, are less likely to facilitate the satisfaction of basic human (organismic) needs, which functions as the "input" for all individuals" optimal functioning. Consistent with this, Kasser and Ahuvia (2002) and Vansteenkiste, Duriez, et al. (2006) found that extrinsic, relative to intrinsic, goal pursuit was associated with lower well-being and more internal distress among a group of business students (see also Srivastava, Locke, \& Bartol, 2001), even though extrinsic goals tend to be heavily emphasized in business environments (Holland, 1985).

More recently, Vansteenkiste and colleagues (see Vansteenkiste, Lens, \& Deci, 2006, for an overview) extended this work by examining the impact on participants' learning and performance of experimentally framing a learning activity in terms of an intrinsic versus extrinsic goal attainment. Thus, instead of treating intrinsic versus extrinsic goal contents as an individual-differences characteristic, they studied goal contents from a social contextual viewpoint - that is, by considering the extent to which goal contents are promoted by the social environment. On the basis of SDT, it was reasoned that intrinsic goals concern learning in the service of inherent psychological needs and growth tendencies and, hence, should promote learning. In contrast, extrinsic goals, with their focus on external indicators of worth, are more likely to distract people from the learning activity and, hence, should result in poorer learning. In a series of experimental field studies, it was found that framing a learning activity in terms of the attainment of an intrinsic goal enhanced conceptual learning, performance, and persistence compared to (a) an extrinsic goal-framing condition (Vansteenkiste, Simons, Lens, Sheldon, \& Deci, 2004), (b) a double (i.e., intrinsic plus extrinsic) goal-framing condition (Vansteenkiste, Simons, Lens, Soenens, et al., 2004), and (c) a no-goal control condition (Vansteenkiste, Simons, Soenens, \& Lens, 2004), whereas extrinsic goal framing undermined learning and performance both compared to the double goal-framing condition and the no-goal control condition. These findings were replicated among 11-12-year-old children (Vansteenkiste et al., 2005).

Although research has shown that experimental extrinsic, relative to intrinsic, goal framing undermines conceptual learning and performance, the question should be raised regarding whether these negative effects also apply to individuals who are, by themselves, more strongly oriented toward the pursuit of extrinsic than intrinsic goals. On the basis of the match perspective, it can be suggested that the negative effects of extrinsic goal framing are limited to learners who value intrinsic goals over extrinsic goals and that the overall enhancement of learning and persistence in the intrinsic goal conditions of the studies reviewed earlier was carried primarily by those learners whose goal orientation was more intrinsic than extrinsic. To better understand the hypothesis derived from the match perspective, we describe this perspective in more detail below.

\section{The Match Perspective}

The match perspective posits that the effect of the content of people's goal pursuits depends on the match or fit between the goal content and the type of goals that are emphasized in the interpersonal environment (Sagiv \& Schwartz, 2000). The theme of match or fit between the person and the environment is represented in a broad array of fields in psychological research, as diverse as social (e.g., Sagiv \& Schwartz, 2000), educational (e.g., Harackiewicz \& Elliot, 1998), organizational (e.g., Edwards, 1991; Meglino, Ravlin, \& Adkins, 1989; Ostroff, 1993; Ostroff, Shin, \& Kinicki, 2005; Pervin, 1968), developmental (e.g., Eccles, Lord, \& Midgley, 1991), and sport (e.g., Amiot, Vallerand, \& Blanchard, 2006) psychology. However, most of this work, except for Sagiv and Schwartz (2000), concerned the examination of a personenvironment match in goals other than the intrinsic-extrinsic goal dimension outlined within SDT. Hence, the present research extends previous work on the match perspective by examining whether the match perspective also holds with regard to the theoretical distinction between intrinsic and extrinsic goals, which has received increasing empirical attention in the educational and motivational literature (see Vansteenkiste, Lens, \& Deci, 2006).

Sagiv and Schwartz (2000) claimed that the negative effect of extrinsic, relative to intrinsic, goal pursuits should be reversed when people find themselves in an extrinsic goal context that emphasizes extrinsic goals over intrinsic goals. They provided three related mechanisms to explain this matching effect. First, social environments that match individuals' goals afford better opportunities to translate one's goals into plans, to carry out one's plans, and, hence, to attain one's goals. Second, when people reject the goals and values that prevail in their environment, they may be ignored, ostracized, or sanctioned, while acceptance of the socially promoted goals is likely to elicit social support and reinforcement, which should promote well-being. Third, the internal conflict that people experience when their earlier acquired goals are in conflict with the goals supported by the social environment is also likely to forestall their well-being.

In line with the match perspective, Sagiv and Schwartz (2000), relying on Schwartz's (1992) value classification, showed in a sample of psychology and business students that the effect of valuing extrinsic goals over intrinsic goals on well-being interacted with participants' study environment. Business students who valued extrinsic goals over intrinsic goals reported higher psychological well-being, while psychology students reported more optimal functioning when they valued intrinsic goals over extrinsic goals.

The present study builds on the work of Sagiv and Schwartz (2000), because it examined whether the experimental induction of an intrinsic versus an extrinsic goal prior to engagement in a learning activity differentially affected learning and performance among children who valued intrinsic goals over extrinsic goals versus children who valued extrinsic goals over intrinsic goals. 
Although the three mechanisms reviewed above are primarily relevant for the issue of well-being, the third mechanism (i.e., internal conflict) might also help us to understand the effects of match versus mismatch on learning and performance. That is, the third mechanism suggests that when learners are placed in a mismatch condition, they face an internal conflict because their own personal goal orientation does not fit with the goal that is experimentally induced. Two groups of learners are likely to face a mismatch and, hence, to experience internal conflict: (a) learners who personally value extrinsic goals over intrinsic goals and are placed in an intrinsic goal condition, and (b) learners who personally value intrinsic goals over extrinsic goals and are placed in an extrinsic goal condition. The experience of internal conflict might result in a less concentrated and less task-involved approach to the learning activity and, hence, help to explain why the mismatch, versus the match, conditions should undermine performance and persistence.

In addition to examining whether a match between one's personal goal orientation and the goal-framing condition yields more optimal learning, we also examined whether being placed in an experimental goal-framing condition that matches with one's spontaneous perception of the learning activity promotes optimal learning. Specifically, herein we suggest that there exists considerable variation in the type of goals people might want to attain through the same activity. Whereas some individuals perceive an activity (e.g., exercising) as serving the attainment of an intrinsic goal (e.g., health), others might perceive the same activity as serving the attainment of an extrinsic goal (e.g., physical appearance). Therefore, we examined whether the effect of experimental intrinsic versus extrinsic goal framing would also hold for individuals who perceived the activity as serving an extrinsic goal. This is an important question, as it might be argued that most of the topics (e.g., healthy lifestyles, recycling) that participants learned about in previous studies on intrinsic versus extrinsic goal framing (e.g., Vansteenkiste, Simons, Lens, Sheldon, \& Deci, 2004; Vansteenkiste, Simons, et al., 2005) had, on average, a rather intrinsic goal character. Hence, the observed positive effects of intrinsic goal framing when participants learned about these topics might have been due to the match between the induced intrinsic goal and the spontaneous perception of the activity as serving an intrinsic goal. The present study aimed to shed more light on this possible confound by directly examining whether the match between one's personal perception of the activity as serving an intrinsic or an extrinsic goal and the experimentally induced intrinsic versus extrinsic goal would promote learning, performance, and persistence.

\section{The Present Research}

The present experimental field study aimed to further examine the conflicting hypotheses that can be derived from SDT and the match perspective. Participants in the current project were fifthand sixth-grade children who learned more about a prosocial organization that is very well known in Flanders (Belgium) - that is, the Father Damian Foundation. This foundation financially supports individuals with tuberculosis. Children in the intrinsic goal condition were told that reading about and supporting the Father Damian Foundation could be important to help patients with tuberculosis (i.e., community contribution), whereas partici- pants in the extrinsic goal condition were told that reading about and supporting the Father Damian Foundation could be important to make a good impression on their peers, parents, and teachers (i.e., social recognition).

One week prior to their participation in the field experiment, children provided answers to two sets of questions. First, they indicated to what extent they personally valued the intrinsic goal of community contribution and the extrinsic goal of social recognition. Second, they indicated whether, if they were asked to participate in activities that support the Father Damian Foundation, they would do so to make a good impression on others and to gain social recognition (extrinsic goal) or to help people with tuberculosis (intrinsic goal). Thus, prior to their participation in the actual experiment, children indicated whether they saw a potential engagement in the Father Damian Foundation, which was also the topic they would learn about during the experimental phase, as serving the attainment of an intrinsic or an extrinsic goal.

One week after we assessed participants' personal intrinsic versus extrinsic goal orientation and their intrinsic versus extrinsic goal perception of the activity, children were randomly assigned to an intrinsic or extrinsic goal-framing condition that either matched or mismatched their personal goal orientation and their personal task perception. Because the intrinsic and extrinsic goals prevailing in the environment were not subjectively assessed but instead were experimentally induced, the present research allowed for a direct examination of the effect of an objective personenvironment match (Ostroff et al., 2005).

Dependent variables in the current project included the assessment of participants' autonomous motivation for engaging in the learning activity as well as their graded performance and freechoice persistence. Autonomous motivation refers to a willing or volitional engagement in learning, because the learning activity is perceived as interesting and enjoyable (intrinsic motivation) and as personally meaningful (identified motivation). Two types of performance were assessed-that is, conceptual learning, which requires deep and thoughtful processing of information and a more creative and integrative solution, and rote learning, which only requires a superficial engagement in the learning and has a more straightforward or rote path to the solution (literal memorization of factual information is sufficient; Entwistle \& Entwistle, 1991; Grolnick \& Ryan, 1987). Although past research has shown that extrinsic goal framing yields a debilitating effect on conceptual learning, such negative effects were not observed for rote learning (Vansteenkiste et al., 2005). Presumably, extrinsic goal framing results in an attentional shift away from the activity at hand, which precludes a thoughtful elaboration of the learning material, which is necessary for conceptual learning to take place.

The following three hypotheses were tested. First, on the basis of SDT, it was expected that intrinsic vs. extrinsic goal framing would predict more autonomous motivation, more conceptual (but not rote) learning, and more persistence for all learners - that is, for both intrinsic and extrinsic goal-oriented individuals. Second, on the basis of the match perspective, it was examined whether intrinsic goal-oriented individuals would benefit from intrinsic goal framing (i.e., match) and whether their learning would be undermined in the extrinsic goal condition (i.e., mismatch). Similarly, from this perspective, it can be anticipated that extrinsic goal-oriented individuals should benefit from extrinsic goal framing (i.e., match), whereas their learning should be forestalled in the 
intrinsic goal condition (i.e., mismatch). Technically speaking, we examined whether the main effect of intrinsic versus extrinsic goal framing would be moderated by participants' personal intrinsic versus extrinsic goal orientation (i.e., interaction effect).

Third, on the basis of the match perspective, it was examined whether participants who saw the activity as serving an intrinsic goal would benefit from intrinsic goal framing (i.e., match), whereas their learning would be undermined in the extrinsic goal framing condition (i.e., mismatch). Similarly, from this perspective, it was expected that participants who saw the activity as serving an extrinsic goal would display more optimal learning when placed in the extrinsic goal framing condition (i.e., match), whereas their learning would be undermined in the intrinsic goal framing condition (i.e., mismatch). Accordingly, we examined whether the predicted main effect of contextual intrinsic versus extrinsic goal framing would be moderated by individuals' perception of the activity as serving an extrinsic or an intrinsic goal.

\section{Method}

\section{Participants and Procedure}

Seventy female and 68 male fifth- and sixth-grade children $(N=$ 138) participated in the study. In a first stage, which took place 1 week prior to the actual experiment, participants filled out questionnaires assessing their personal intrinsic versus extrinsic goal orientations and their perception of the intrinsic versus extrinsic goal character of the activity they would participate in about 1 week later (see below). The second stage took place during a religious meeting-preparing Roman Catholic children for their confirmation - in which participants were given a text about the Father Damian Foundation that they would have to read anyway.

The study took place in schools that are located in the town where Father Damian was born. Father Damian is a very wellknown and popular public figure in this neighborhood, and several activities are organized yearly in this area to support the foundation and to remember Father Damian. For this reason, it is very normal that children at a relatively young age learn more about Father Damian and the Father Damian Foundation. The present experiment took place at the time the Damian campaign was organized.

Within each class, children were randomly assigned to an intrinsic or an extrinsic goal condition (cell sample sizes of 68 and 70 , respectively) and read an instruction sheet (in Dutch) prior to studying the Father Damian text. We made sure that instruction sheets were of similar length so that participants would be unaware that they had received different instructions. The instruction sheets were turned in at the end of the session, after the children had written their names on them. The instructions included one of two different goal inductions for the forthcoming learning task. In the intrinsic going-framing condition, the children read that by learning more about the Father Damian Foundation, they could learn how to help individuals with tuberculosis (i.e., "Doing your best to read the text about the Father Damian Foundation might help you to know more about how you could help people with tuberculosis. By supporting the Father Damian Foundation you can help to save the lives of tuberculosis patients"), which constituted the intrinsic goal of community contribution (Kasser \& Ryan, 1996). In the extrinsic goal-framing condition, they read that learning more about and supporting the Father Damian Foundation would help them to attain the goal of being admired by others (i.e., "Doing your best to read the text about the Father Damian Foundation might help you to collect a lot of money and hence be admired by others. Thus, supporting the Father Damian Foundation is important to gain the social recognition of others"), which reflected the extrinsic goal of social recognition (Kasser \& Ryan, 1996).

After studying the text, participants completed a questionnaire that assessed their degree of autonomous motivation for reading the text. Immediately following the completion of this questionnaire, participants were tested on their knowledge concerning the text (20 min). Subsequently, they were told that they could take home a comic book (i.e., drawing) about the Father Damian Foundation if they wanted to.

\section{Measures}

Personal intrinsic and extrinsic goal orientation. Participants' intrinsic and extrinsic goal orientations are typically assessed with Kasser and Ryan's (1996) Aspiration Index. In previous research among Dutch-speaking university (Vansteenkiste, Duriez, et al., 2006) and high school students (Duriez, Vansteenkiste, Soenens, \& De Witte, 2007), we have successfully developed a shortened Dutch version of this questionnaire. In the current study, participants filled out an adapted (i.e., simplified) child version of this shortened Dutch version of the Aspiration Index. They recorded how much they valued both types of goals by circling a number on a 5-point Likert scale ranging from 1 (not important at all) to 5 (very important). We only used the data for the intrinsic goal of community contribution (four items) and the extrinsic goal of social recognition (four items), as these represent the two goals that were manipulated in the present research. ${ }^{2}$

An exploratory factor analysis using promax rotation indicated that two factors needed to be retained (eigenvalues $=3.52$ and 2.73 ), explaining $78 \%$ of the variance across the four community contribution items (e.g., "Helping other people") and the four social recognition items (e.g., "I will be admired by other people"). Each of the items had a minimal loading of .34. We constructed social recognition and community contribution scores by averaging the social recognition and community contribution items, respectively. Internal consistencies of the community contribution and social recognition scores were .81 and .94 , respectively. These analyses provide evidence for the structure of our goal measure and indicate that children's personal valuation of community contribution and social recognition can be reliably measured.

Intrinsic and extrinsic task perception. Intrinsic and extrinsic task perception were assessed with two 1-item measures. Partici-

\footnotetext{
${ }^{2}$ We believe that only using the goals of community contribution and social recognition, instead of the full Intrinsic and Extrinsic Goals scales, allows for a more accurate testing of the match hypothesis. This is because the match between the personally held goals (e.g., community contribution or social recognition) and the contextually promoted goal (e.g., community contribution or social recognition) would be strongest if one only retained the intrinsic goal of community contribution and the extrinsic goal of social recognition. The inclusion of the other intrinsic and extrinsic goals in the personal goal orientation measure would result in a poorer match between the personally held and contextually promoted goals and, as a result, would represent a methodologically weaker way of testing the match hypothesis.
} 
pants were asked how important they found it to support the Father Damian Foundation to achieve the intrinsic goal of community contribution (i.e., "I prefer to support the Father Damian Project because it gives me the opportunity to help other people") or the extrinsic goal of social recognition (i.e., "I prefer to support the Father Damian Project because of the good impression it makes on other people"). We focused on these two goals only because they represent the two goals that were manipulated during the actual experiment. On both items, participants indicated their agreement on a 10-point Likert scale that varied from 1 (completely disagree) to 10 (completely agree).

Autonomous motivation. Consistent with SDT and previous experimental studies among children (Vansteenkiste et al., 2005), autonomous motivation was measured by the extent to which participants found the reading material enjoyable and interesting (e.g., "I read the text because I found it very interesting"; intrinsic motivation; four items; $\alpha=.91$ ) and personally relevant and meaningful (e.g., "I read this text because the content is personally meaningful to me"; identified regulation; four items; $\alpha=.81$ ). Participants indicated their agreement with each of the items by circling a number between 1 (completely disagree) and 5 (completely agree). As in previous research (e.g., Vansteenkiste, Lens, Dewitte, De Witte, \& Deci, 2004), we created a composite score of autonomous motivation by averaging the scores on these two subscales $(\alpha=.82)$.

Test performance. Two aspects of learning were assessedthat is, conceptual and rote learning. Five short questions tapped rote learning. To answer these questions, children needed to fill in one single word that was specifically mentioned in the text on Father Damian. As a result, scale scores for rote learning varied between 1 and 5. Concerning conceptual learning, the children were given three problems, which were scored on a scale varying from 1 (very bad) to 15 (very good). The first two conceptual problems were as follows: "Provide three examples that illustrate that Father Damian is a 'stubborn stayer"" (4 points), and "Provide three reasons for the beatification of Father Damian" (3 points). The third problem was a crossword puzzle, which required children to fill in eight words (8 points). Each of the crossword questions tapped a deeper understanding of the learning material, as the answer to the question was not mentioned in the text but needed to be derived from it by meaningful linking of different pieces of information. These problems were created by the teacher and had been used in previous years to test children's knowledge with respect to Father Damian. Therefore, the test was ecologically valid, and the children would have had to respond to the questions even if they had not been involved in the study (see Vansteenkiste et al., 2005, for a similar methodology). The regular teacher and one independent and trained rater, who were both blind to the nature of the study, determined whether the answers to these rote and conceptual learning questions were correct (1) or incorrect (0). There was perfect agreement between the raters scoring each question, as indexed by a perfect Pearson correlation between the two sets of rating scores.

Persistence. Participants were offered the opportunity to take home a comic book on Father Damian so that they could learn more about him and his foundation. The instructor registered who took home a comic book. In total, 75 participants (54\%) did so. This constitutes a behavioral measure of free-choice persistence, as it represents the tendency to display continued interest in the learning material once the learning task is terminated.

\section{Results}

\section{Preliminary Analyses}

One-sample $t$ testing, $t(137)=7.90, p<.001$ indicated that, as a whole, the children valued the intrinsic goal of community contribution $(M=3.34, S D=1.11)$ more strongly than the extrinsic goal of social recognition $(M=2.43, S D=0.88)$, a finding that replicates previous work on intrinsic versus extrinsic goals among undergraduate students (e.g., Grouzet et al., 2006). To examine what percentage of children valued social recognition over community contribution and what percentage of children valued community contribution over social recognition, we subtracted participants' social recognition score from their community contribution score. Then we dichotomized this difference measure by assigning all participants who had a negative difference score (indicating that they valued social recognition over community contribution) a score of -1 and by assigning all participants who had a positive difference score (indicating that they valued community contribution over social recognition) a score of 1 . In total, $30 \%$ of the participants $(n=41)$ valued social recognition over community contribution, and $70 \%$ of the participants $(n=97)$ valued community contribution over social recognition. In the remainder of this article, the former group is referred to as the extrinsic goal-oriented participants, whereas the latter group is referred to as the intrinsic goal-oriented participants.

Next, we created four different groups by crossing contextual intrinsic versus extrinsic goal framing with the dichotomized personal intrinsic versus extrinsic goal orientation measure. Then we performed a chi-square test on the percentages of intrinsic and extrinsic goal-oriented individuals who were placed in the intrinsic and extrinsic goal-framing conditions. The purpose of this analysis was to examine whether intrinsic and extrinsic goal-oriented participants were equally distributed over the intrinsic and extrinsic goal-framing conditions. The percentages and exact cell sizes can be found in Table 1. The chi-square test turned out to be nonsignificant, $\chi^{2}(1, N=138)=0.85$, indicating that the intrinsic and extrinsic goal-framing conditions consisted of an equal percentage of intrinsic and extrinsic goal-oriented individuals.

Table 1

Frequencies and Cell Sizes per Condition (Contextual Goal Framing $\times$ Personal Goal Orientation)

\begin{tabular}{|c|c|c|c|}
\hline \multirow{2}{*}{$\begin{array}{l}\text { Contextual } \\
\text { goal framing }\end{array}$} & \multicolumn{2}{|c|}{ Personal goal orientation } & \multirow[b]{2}{*}{ Row total } \\
\hline & Intrinsic & Extrinsic & \\
\hline \multicolumn{4}{|l|}{ Intrinsic } \\
\hline$\%$ & 71 & 29 & 51 \\
\hline$n$ & 50 & 20 & 70 \\
\hline \multicolumn{4}{|l|}{ Extrinsic } \\
\hline$\%$ & 69 & 31 & 49 \\
\hline$n$ & 47 & 21 & 68 \\
\hline \multicolumn{4}{|l|}{ Column total } \\
\hline$\%$ & 70 & 30 & 100 \\
\hline$n$ & 97 & 41 & 138 \\
\hline
\end{tabular}


A similar set of analyses was performed on participants' intrinsic versus extrinsic task perception. One-sample $t$ testing, $t(137)=$ $8.13, p<.001$, indicated that, on average, children were more likely to see their participation in the Father Damian Foundation as serving the intrinsic goal of community contribution $(M=6.54$, $S D=1.80$ ) than as serving the extrinsic goal of social recognition $(M=4.01, S D=2.23)$. To examine what percentage of children perceived participation in the Father Damian Foundation as more strongly serving community contribution than social recognition and what percentage of children saw their participation as more strongly serving social recognition than community contribution, we subtracted the former scale from the latter. Then we dichotomized this continuous difference scale by assigning a score of -1 if the participants had a negative difference score (indicating that they saw their participation as more strongly serving social recognition than community contribution) and assigning a score of 1 if participants had a positive difference score (indicating that they saw their participation as more strongly serving community contribution than social recognition). In total, $33 \%$ of the participants $(n=46)$ saw their participation in the Father Damian Foundation as serving social recognition more strongly than community contribution, whereas $67 \%$ of the participants $(n=92)$ saw their participation as more strongly serving community contribution.

Next, we created four groups by crossing intrinsic versus extrinsic goal framing with the constructed dichotomous intrinsic versus extrinsic task perception measure. Percentages and exact numbers of participants per group can be found in Table 2. Again, we performed a chi-square test with the purpose of examining whether individuals who perceived the task as intrinsically or extrinsically goal oriented were equally distributed across the two experimental conditions. The chi-square test turned out to be nonsignificant, $\chi^{2}(1, N=138)=0.72$, indicating that both groups of participants were equally distributed over the intrinsic and extrinsic goal-framing conditions.

In sum, these preliminary analyses indicated (a) that the sample of participants was quite heterogeneous in terms of both its intrinsic-extrinsic goal profile and its perception of the Father Damian Foundation as serving an intrinsic or extrinsic goal; (b) that the extrinsic and intrinsic goal-oriented participants, as well as the participants who saw the Father Damian Foundation as primarily serving an intrinsic goal or an extrinsic goal, were equally

Table 2

Frequencies and Cell Sizes per Condition (Contextual Goal Framing $\times$ Goal Activity Perception)

\begin{tabular}{lccc}
\hline & \multicolumn{2}{c}{ Goal activity perception } & \\
\cline { 2 - 3 } $\begin{array}{l}\text { Contextual } \\
\text { goal framing }\end{array}$ & Intrinsic & Extrinsic & Row total \\
\hline Intrinsic & & & \\
$\quad \%$ & 69 & 31 & 51 \\
$n$ & 48 & 22 & 70 \\
Extrinsic & 65 & 35 & 49 \\
$\quad \%$ & 44 & 24 & 68 \\
$n$ & & & \\
Columnn & 67 & 33 & 100 \\
$\quad$ total & 92 & 46 & 138 \\
$\quad \%$ & & & \\
$n$ & & & \\
\hline
\end{tabular}

distributed across the two experimental conditions; and (c) that each of the created groups, which represented a combination of contextual goal framing with either participants' personal goal orientation or their task perception, consisted of a sufficiently large number of participants (more than 20). Thus, these preliminary analyses indicated that we could proceed with the testing of our primary hypotheses.

Finally, to examine possible gender effects, we performed an independent-sample $t$ test including autonomous motivation, performance, and persistence as dependent variables. Girls $(M=$ $2.94, S D=0.48)$ were found to be more autonomously motivated than boys $(M=2.75, S D=0.51), t(136)=2.28, p<.05$, but no gender differences were found with respect to conceptual learning, rote learning, and persistence. Hence, we controlled for gender effects in subsequent analyses when predicting autonomous motivation.

\section{Primary Analyses}

The means and standard deviations for all variables across conditions, as well as the correlations between each pair of variables, are presented in Table 3. We examined our central hypotheses by performing two sets of regression analyses, one involving the difference score of intrinsic versus extrinsic goal orientations as a moderator, and one involving the difference score of intrinsic versus extrinsic activity perceptions as a moderator of experimental intrinsic versus extrinsic goal framing. We conducted multiple regression analyses to examine the effects on the continuous outcomes of autonomous motivation and both types of performance, but we made use of logistic regression analyses to examine the effects on the categorical outcome of persistence. In these primary analyses, we preferred to use the continuous (instead of the dichotomous) difference measures of intrinsic versus extrinsic goal orientations and intrinsic versus extrinsic goal task perception to increase statistical power.

In the first series of regressions, experimental intrinsic versus extrinsic goal framing, the continuous difference measure of intrinsic versus extrinsic goal orientation and the goal framing $\times$ goal orientation interaction measure were entered in the regression equation. Experimental goal framing and goal orientation were centered, and the interaction term was calculated as the product of these centered scores. Multiple regression analyses indicated that experimental intrinsic, relative to extrinsic, goal framing positively predicted autonomous motivation $(\beta=.43, p<.001)$ and conceptual learning $(\beta=.31, p<.001)$ but was unrelated to rote learning $(\beta=.13, n s)$. Intrinsic versus extrinsic goal orientation positively predicted autonomous motivation $(\beta=.17, p<.05)$ but was unrelated to conceptual learning $(\beta=.01, n s)$ and rote learning $(\beta=-.05, n s)$.

On the basis of the match perspective, the main effect of experimental goal framing should be altered depending on individuals' personal goal orientations. However, the interactions between experimental goal framing and goal orientation in the prediction of autonomous motivation $(\beta=-.08, n s)$, conceptual learning $(\beta=-.01, n s)$, and rote learning $(\beta=.06, n s)$ were not significant. Concerning persistence, a logistic regression analysis pointed out that the three predictors yielded an overall effect on persistence (dummy coded as lack of persistence $=0$ and persistence $=1), \chi^{2}(3, N=138)=25.58, p<.001$. Persistence was 
Table 3

Means, Standard Deviations, and Intercorrelations Between Independent and Dependent Variables

\begin{tabular}{|c|c|c|c|c|c|c|c|c|c|}
\hline Variable & $M$ & $S D$ & Observed Range & 1 & 2 & 3 & 4 & 5 & 6 \\
\hline 1. Personal intrinsic vs. extrinsic goal & 0.91 & 1.36 & -3 to 4 & - & & & & & \\
\hline 2. Intrinsic vs. extrinsic task perception & 2.53 & 3.66 & -6 to 8 & $.60^{* *}$ & - & & & & \\
\hline 3. Autonomous motivation & 2.92 & 0.50 & 2 to 4 & $.16^{*}$ & .15 & - & & & \\
\hline 4. Conceptual learning & 9.41 & 2.39 & 3 to 15 & .02 & .11 & $.22^{*}$ & - & & \\
\hline 5. Rote learning & 3.78 & 0.81 & 2 to 5 & -.06 & .14 & .08 & $.45^{* *}$ & - & \\
\hline 6. Persistence & 0.54 & 0.50 & 0 to 1 & .10 & $.22^{*}$ & $.22^{*}$ & $.47^{* * *}$ & $.32^{* * *}$ & - \\
\hline
\end{tabular}

${ }^{*} p<.05 .{ }^{* *} p<.01$.

significantly predicted by placement in the experimental intrinsic goal-framing condition (odds ratio $[\mathrm{OR}]=0.17, p<.001$ ) but not by the personal goal orientation measure $(\mathrm{OR}=1.22, n s)$ nor by the interaction between experimental goal framing and the personal goal orientation measure $(\mathrm{OR}=0.94, n s)$. Together, the predictors explained between $2 \%$ and $26 \%$ of the variance in the outcomes.

In a second set of regressions, the three outcomes were regressed on experimental intrinsic versus extrinsic goal framing, the continuous difference measure of intrinsic versus extrinsic activity perception, and the interaction between experimental goal framing and activity perception. Concerning autonomous motivation and performance outcomes, multiple regression analyses indicated that experimental intrinsic versus extrinsic goal framing positively predicted autonomous motivation $(\beta=.42, p<.001)$ and conceptual learning $(\beta=.34, p<.001)$ but was unrelated to rote learning $(\beta=.09)$. Intrinsic versus extrinsic activity perception was unrelated to autonomous motivation $(\beta=.11, n s)$, conceptual learning $(\beta=.10, n s)$, and rote learning $(\beta=.13, n s)$. Most important, the interaction between experimental goal framing and activity perception did not reach significance (autonomous motivation, $\beta=.14, n s$; conceptual learning, $\beta=-.05$, $n s$; and rote learning, $\beta=.05, n s)$. Concerning persistence, a logistic regression analysis indicated that the three predictors yielded an overall effect, $\chi^{2}(3, N=138)=30.69, p<.001$. Persistence was significantly predicted by placement in the experimental intrinsic goal framing condition $(\mathrm{OR}=0.14, p<.001)$ and by the presence of an intrinsic activity perception $(\mathrm{OR}=1.15, p<.01)$ but not by the interaction between experimental goal framing and the activity perception measure $(\mathrm{OR}=0.93, n s)$. Together, the predictors explained between $4 \%$ and $27 \%$ of the variance in the outcomes. ${ }^{3}$

\section{Discussion}

The present research examined conflicting hypotheses derived from SDT and the match perspective regarding the impact of intrinsic versus extrinsic goal framing on learning and performance. This research extends previous work in this area by using an experimental instead of a correlational design, by focusing on learning and performance instead of well-being as outcomes, and by examining the possible moderating role of two types of individual-differences variables (i.e., intrinsic vs. extrinsic goal orientation and intrinsic vs. extrinsic activity perception) in the relation between intrinsic versus extrinsic goal framing and learning and performance. Several important findings emerged.
Several previous studies among fifth- to sixth-grade children, high school students, and college students have shown that the experimental induction of intrinsic goals promotes better conceptual learning and persistence compared to the induction of extrinsic goals (see Vansteenkiste, Lens, \& Deci, 2006). The present research fully replicated previous studies by showing that extrinsic, relative to intrinsic, goal framing undermined participants' selfreported task enjoyment and personal valuation of the activity (i.e., autonomous motivation) and forestalled their conceptual performance and persistence. The reason for these effects is probably that inducing an extrinsic goal creates an outward orientation by drawing individuals' attention toward the external signs of worth. This attentional shift precludes the possibility of task-absorbed engagement in the activity, so that participants fail to enjoy the learning activity and perform less well (Vansteenkiste, Lens, \& Deci, 2006). As in previous experimental work (Vansteenkiste et al., 2005), the negative effect of extrinsic goal framing did not emerge for rote learning. Apparently, extrinsic goal framing prompts some motivated learning; however, the learning activity is approached in a more rigid and narrow-minded fashion, because the learning is primarily oriented toward attaining the extrinsic goals. Such a rigid and restricted approach is sufficient to promote memorization of learning material but interferes with an in-depth processing of the contents.

Beyond replicating past results, the main contribution of the present work was to examine whether the effects of contextual goal framing depended on participants' own goal preferences. Vansteenkiste, Simons, Lens, Sheldon, and Deci (2004) found that extrinsic (vs. intrinsic) goal framing undermined optimal learning among a group of business students, but they did not include assessments of individuals' own goal orientations prior to participation in the experiment. As such, the authors had no opportunity to directly examine whether goal framing interacted with personal goal orientations. In particular, research to date has not directly tested the possibility that the observed negative effects of extrinsic goal framing would be reversed for extrinsic goal-oriented indi-

\footnotetext{
${ }^{3}$ As recommended by one of the anonymous reviewers, we conducted a power analysis. A post hoc power analysis indicated that with the current sample size of $138, \alpha=.05$, three used predictors, and $R^{2}=.20$, the current set of regression analyses yielded sufficient power (i.e., 1.00) to detect significant effects. An a priori power analysis indicated that with a desired power of .80, an alpha level of . 05 , an estimated effect size of .25 , and a total of three predictors, we would need to sample at least 48 participants. The current sample size meets this requirement amply.
} 
viduals, as would be predicted by the match perspective. The present research addressed exactly this question.

On average, participants in the present sample were found to more strongly value the intrinsic goal of community contribution than the extrinsic goal of social recognition, a finding that is in line with various previous studies (e.g., Grouzet et al., 2006). In spite of this, $30 \%$ of the present sample attached higher importance to social recognition than to community contribution, which suggests that the current sample was relatively heterogeneous in terms of its intrinsic-extrinsic goal profile. Furthermore, the extrinsic goaloriented individuals appeared to be equally distributed across the intrinsic and extrinsic goal-framing conditions, and, thus, participants appeared to be randomly dispersed across the experimental conditions. Interaction analyses between contextual goal framing and personal goal orientation indicated that the effect of contextual intrinsic versus extrinsic goal framing did not depend on participants' personal intrinsic versus extrinsic goal orientation. Thus, the match perspective could not be confirmed, as both intrinsic and extrinsic goal-oriented individuals benefited from being placed in an intrinsic goal condition. Presumably, the shift in attention that we caused by inducing an extrinsic goal negatively affected both intrinsic and extrinsic goal-oriented individuals. Referring to an extrinsic goal elicits self-worth concerns and distracts all participants' attention from the activity at hand, which undermines optimal learning (Vansteenkiste, Lens, \& Deci, 2006). In contrast, learning in the service of intrinsic goals is more consistent with people's basic psychological needs, which should promote more optimal learning for all individuals (Deci \& Ryan, 2000; Kasser, 2002).

The match perspective was also tested in a second way. We suggest that there exists considerable variability in the type of goals the same activity might serve. Whereas some individuals might perceive an activity as serving the attainment of intrinsic goals, other individuals would see the same activity as a means toward extrinsic goal attainment. A possible confound in previous work on intrinsic versus extrinsic goal framing is that the selected activities (e.g., reading a text on recycling) might, on average, be perceived as serving an intrinsic goal. When individuals are subsequently instructed that the activity serves an intrinsic goal, they find themselves in a match situation, whereas individuals who are told that the activity serves an extrinsic goal find themselves in a mismatch situation. The induced extrinsic goal might even be seen as meaningless and the instructions as unbelievable, because they conflict with participants' spontaneous goal perception of the activity.

To eliminate this possible confound, we obtained direct assessments of the type of goals individuals pursued through supporting the Father Damian Foundation, the topic they would read about during the experimental phase. Although children saw the activity, on average, as standing more strongly in the service of intrinsic than extrinsic goal attainment, there was substantial variation in their perceptions. That is, one third of the children saw the activity as instrumental for extrinsic goal attainment (i.e., social recognition), whereas the other two thirds saw the activity as instrumental for intrinsic goal attainment (i.e., community contribution). We found that the effect of intrinsic versus extrinsic goal framing was not altered for individuals who spontaneously saw the activity as serving an extrinsic goal. All individuals, regardless of their activity perception, displayed more optimal learning in the intrinsic, compared to the extrinsic, goal-framing condition.
Another set of findings that deserves some discussion is the observation that individuals' intrinsic versus extrinsic goal profiles and intrinsic versus extrinsic activity perception had smaller effects on their motivation, learning, and performance compared to the effects of contextual goal framing. Although these results need to be replicated in future research, we provide two explanations. First, the individual goal measures were assessed 1 week prior to children's participation in the experiment, whereas children read the instructions on intrinsic versus extrinsic goal framing a few minutes before reading the text. Because of this different time lag, it seems logical that contextual goal framing yielded stronger effects compared to the two individual-differences measures.

A second interpretation deals with the level of generality at which the independent variables can be situated. That is, within the hierarchical model of motivation (Vallerand, 1997), a global (i.e., personality), a domain-bounded (i.e., in a particular life domain), and a situational (i.e., for a specific task) level of motivation are distinguished. Clearly, the present study examined participants' learning and performance on a specific task-that is, at the situational level of motivation. Whereas participants' intrinsic and extrinsic goal profiles reflect their global endorsement of goals, contextual goal framing represents a manipulation at the situational level, because the presented goals are directly linked to the learning activity itself. Hence, it seems logical that contextual goal framing is a better predictor of situation-bounded outcomes than participants' goal profiles, because the former are situated at a similar level of motivation, whereas the latter are not (see also Roberts \& Pomerantz, 2004, for a similar reasoning).

Does the current set of results imply that all types of goal-match situations will invariantly fail to predict optimal performance? We do not think so. Researchers have focused on very different types of goals and psychological constructs to examine the match hypothesis (see Amiot et al., 2006; Bianco et al., 2003; Harackiewicz \& Elliot, 1998). The present research differs from that work because it focuses on the concepts of intrinsic versus extrinsic goals. To address the question of whether particular goals will promote optimal functioning, SDT suggests that researchers evaluate the functional significance of these goals by asking how they are dynamically related to basic psychological need satisfaction. If the pursuit or induction of particular goals fails to promote basic need satisfaction, a match situation is unlikely to override the negative effects associated with thwarted need satisfaction. Because extrinsic goal induction is, on average, unrelated or even negatively related to basic need satisfaction, these goals should result in diminished learning, even when individuals strongly value these goals, as convincingly shown in the present research SDT's position regarding the motivational impact of goals also implies that if particular goals cannot be meaningfully tied to basic need satisfaction, placing the individual in a match situation might predict more optimal functioning. Overall, we believe that more research is needed to carefully examine whether the match hypothesis holds for particular goals, whereas it does not for other types of goals.

\section{Practical Implications}

Previous research (e.g., Kasser \& Ahuvia, 2002; Sagiv \& Schwartz, 2000) investigated whether extrinsically oriented individuals benefit psychologically if their personal goals match with the goals emphasized in their environment. Accordingly, these studies focused on the question of whether a person's goals should 
match with the environmentally promoted goals to predict psychological well-being (i.e., person-environment match). The present study asked a different question: Do socializing agents need to adjust their goal framing in accordance with the goal profiles of the individuals who need to be motivated? Put differently, does the environment need to be accommodated to the persons who are entering it (environment-person match)? If this were true, it would imply that socializing agents first need to gain insight into individuals' personal goal orientations and, subsequently, need to use individualized goal-framing instructions that match the person's goal preference to motivate learning. This seems an extremely difficult task. Luckily, the present experiment indicates that socializing agents' motivational task is much easier: It suffices to induce intrinsic goals when one is trying to promote optimal learning, even if individuals ascribe high importance to extrinsic goals themselves.

Of course, there might be a few boundary conditions to this goal framing effect that could be more thoroughly examined in future research. First, consistent with previous goal research (Locke \& Latham, 1990, 2002), we suggest that a specific, instead of a vague, intrinsic goal is more likely to promote optimal learning (see also Vansteenkiste, Simons, Soenens, \& Lens, 2004). Second, the induced intrinsic goal needs to be realistically and meaningfully connected to the learning activity, so that learners accept the provided intrinsic goal. Past research has shown that providing a goal that is not accepted by participants does not yield the same performance benefits (Locke \& Latham, 1990). Third, although extrinsic goal framing undermines deep-level learning, the current study shows that extrinsic goal framing predicts a similar extent of superficial and shallow learning as intrinsic goal framing (Vansteenkiste et al., 2005).

\section{Limitations and Future Research}

The current project contained a number of shortcomings that might be overcome in future research. First, only one single intrinsic goal and one single extrinsic goal were manipulated. In future studies, researchers might want to examine whether manipulation of other extrinsic goals (e.g., physical appearance, financial success) promotes or undermines optimal learning among individuals who attach importance to these extrinsic goals. Second, it would be interesting to develop a measure of experienced goal conflict versus goal congruence as a check on whether individuals placed in a mismatch condition effectively experience goal conflict, while those placed in a match condition experience goal congruence. Third, future research might sample extrinsically oriented individuals who are older (e.g., business college students) than the ones who participated in the current project to examine whether the match hypothesis could be confirmed (or not) among these older age groups. Finally, the current results might also be examined in different domains from learning and education, including sports and exercising, work, and cross-cultural research. For instance, it would be interesting to examine whether the pursuit of extrinsic goals yields different effects depending on whether one perceives one's coach, one's manager, the organization, or even the culture at large as promoting and reinforcing such goals.

\section{Conclusion}

The results of the present study indicate that framing a learning activity in terms of the attainment of an extrinsic rather than an intrinsic goal undermined task enjoyment, personal valuation of a learning activity, conceptual learning, and persistence, irrespective of individuals' own intrinsic and extrinsic goals or their intrinsic and extrinsic task perception. Hence, these results suggest that instructors, teachers, and other socializing agents might do well to promote intrinsic goals and might downplay the promotion of extrinsic goals to motivate conceptual learning and performance, even for individuals who ascribe high importance to such extrinsic goals.

\section{References}

Amiot, C. E., Vallerand, R. J., \& Blanchard, C. M. (2006). Passion and psychological adjustment: A test of the person-environment fit hypothesis. Personality and Social Psychology Bulletin, 32, 220-229.

Bianco, A. M., Higgins, E. T., \& Klem, A. (2003). How "fun/importance" fit affects performance: Relating implicit theories to instructions. Personality and Social Psychology Bulletin, 29, 1091-1103.

Cohen, P., \& Cohen, J. (1996). Life values and adolescent mental health. Mahwah, NJ: Erlbaum.

Deci, E. L., \& Ryan, R. M. (1985). Intrinsic motivation and selfdetermination in human behavior. New York: Plenum Press.

Deci, E. L., \& Ryan, R. M. (2000). The "what" and the "why" of goal pursuits: Human needs and the self-determination of behavior. Psychological Inquiry, 11, 227-268.

De Witte, H. (2004). Ideological orientations and values. In C. Spielberger (Ed.), Encyclopedia of applied psychology (Vol. 2, pp. 1-10). Amsterdam: Elsevier.

Duriez, B., Vansteenkiste, M., Soenens, B., \& De Witte, H. (2007). The social costs of extrinsic relative to intrinsic goal pursuits: Their relation with social dominance and racial and ethnic prejudice. Journal of Personality, 75, 757-782.

Eccles, J. S., Lord, S., \& Midgley, C. (1991). What are we doing to early adolescents? The impact of educational contexts on early adolescents. American Journal of Education, 99, 521-541.

Edwards, J. R. (1991). Person-job fit: A conceptual integration, literature review, and methodological critique. In C. L. Cooper \& I. T. Robertson (Eds.), International review of industrial and organizational psychology (pp. 283-357). Oxford, England: Wiley.

Entwistle, N., \& Entwistle, A. (1991). Contrasting forms of understanding for degree examinations: The student perspective and its implications. Higher Education, 22, 205-277.

Fromm, E. (1976). To have or to be? New York: Continuum.

Grolnick, W. S., \& Ryan, R. M. (1987). Autonomy in children's learning: An experimental and individual difference investigation. Journal of Personality and Social Psychology, 52, 890-898.

Grouzet, F. M. E., Kasser, T., Ahuvia, A., Dols, J. M. F., Kim, Y., Lau, S., et al. (2006). The structure of goal contents across 15 cultures. Journal of Personality and Social Psychology, 89, 800-816.

Harackiewicz, J. M., \& Elliot, A. J. (1998). The joint effects of target and purpose goals on intrinsic motivation: A mediational analysis. Personality and Social Psychology Bulletin, 24, 675-689.

Holland, J. L. (1985). Making vocational choices: A theory of careers. Englewood Cliffs, NJ: Prentice-Hall.

Kasser, T. (2002). The high price of materialism. Cambridge, MA: MIT Press.

Kasser, T. (2005). Frugality, generosity, and materialism for use in children and adolescents. In K. A. Moore \& L. H. Lippman (Eds.), What do children need to flourish? Conceptualizing and measuring indicators of positive development (pp. 357-374). New York: Springer. 
Kasser, T., \& Ahuvia, A. (2002). Materialistic values and well-being in business students. European Journal of Social Psychology, 32, 137-146.

Kasser, T., \& Ryan, R. M. (1993). A dark side of the American dream: Correlates of financial success as a central life aspiration. Journal of Personality and Social Psychology, 65, 410-422.

Kasser, T., \& Ryan, R. M. (1996). Further examining the American dream: Differential correlates of intrinsic and extrinsic goals. Personality and Social Psychology Bulletin, 22, 280-287.

Kasser, T., Ryan, R. M., Couchman, C. E., \& Sheldon, K. (2004). Materialistic values: Their causes and consequences. In T. Kasser \& A. D. Kanner (Eds.), Psychology and consumer culture: The struggle for a good life in a materialistic world (pp. 11-28). Washington, DC: American Psychological Association.

Lepper, M. R., \& Greene, D. (1978). The hidden costs of reward. Hillsdale, $\mathrm{NJ}$ : Erlbaum.

Locke, E. A., \& Latham, G. P. (1990). Work motivation and satisfaction: Light at the end of the tunnel. Psychological Science, 1, 240-245.

Locke, E. A., \& Latham, G. P. (2002). Building a practically useful theory of goal setting and task motivation. American Psychologist, 57, 705717

Maslow, A. H. (1954). Motivation and personality. New York: Harper.

Meglino, B. H., Ravlin, E. C., \& Adkins, C. L. (1989). A work value approach to corporate culture: A field test of the value congruence process and its relationship to individual outcomes. Journal of Applied Psychology, 74, 424-432.

Nuttin, J. R., \& Lens, W. (1985). Future time perspective and motivation: Theory and research method. Leuven, Belgium/Hillsdale, NJ: Leuven University Press/Erlbaum.

Ostroff, C. (1993). The effects of climate and personal influences on individual behavior and attitudes in organizations. Organizational Behavior and Human Decision Processes, 56, 56-90.

Ostroff, C., Shin, Y., \& Kinicki, A. J. (2005). Multiple perspectives of congruence: Relationships between value congruence and employee attitudes. Journal of Organizational Behavior, 26, 591-623.

Pervin, L. A. (1968). Performance and satisfaction as a function of individual-environment fit. Psychological Bulletin, 69, 56-68.

Roberts, B. W., \& Pomerantz, E. M. (2004). On traits, situations, and their integration: A developmental perspective. Personality and Social Psychology Review, 8, 402-416.

Rohan, M. J. (2000). A rose by a name? The values construct. Personality and Social Psychology Review, 4, 255-277.

Rokeach, M. (1973). The nature of human values. New York: Free Press.

Ryan, R. M., \& Deci, E. L. (2000a). Intrinsic and extrinsic motivations: Classic definitions and new directions. Contemporary Educational Psychology, 25, 54-67.

Ryan, R. M., \& Deci, E. L. (2000b). Self-determination theory and the facilitation of intrinsic motivation, social development, and well-being. American Psychologist, 55, 68-78.

Ryan, R. M., Mims, V., \& Koestner, R. (1983). Relation of reward contingency and interpersonal context to intrinsic motivation: A review and test using cognitive evaluation theory. Journal of Personality and Social Psychology, 45, 736-750.

Ryan, R. M., Sheldon, K. M., Kasser, T., \& Deci, E. L. (1996). All goals were not created equal: An organismic perspective on the nature of goals and their regulation. In P. M. Gollwitzer \& J. A. Bargh (Eds.), The psychology of action: Linking motivation and cognition to behavior ( $\mathrm{pp}$. 7-26). New York: Guilford Press.

Sagiv, L., \& Schwartz, S. H. (2000). Value priorities and subjective well-being: Direct relations and congruity effects. European Journal of Social Psychology, 30, 177-198.

Schneider, B. (1987). E = f (P, B): The road to a radical approach to person-environment fit. Journal of Vocational Behavior, 31, 353-361.

Schwartz, S. H. (1992). Universals in the content and structure of values: Theoretical advances and empirical tests in 20 countries. In M. P. Zanna
(Ed.), Advances in experimental social psychology (Vol. 25, pp. 1-65). New York: Academic Press.

Schwartz, S. H. (1994). Are there universal aspects in the structure and contents of human values? Journal of Social Issues, 50, 19-45.

Schwartz, S. H. (1999). A theory of cultural values and some implications for work. Applied Psychology: An International Review, 48, 23-47.

Srivastava, A., Locke, E. A., \& Bartol, K. M. (2001). Money and subjective well-being: It's not the money, it's the motives. Journal of Personality and Social Psychology, 80, 959-971.

Vallerand, R. (1997). Toward a hierarchical model of intrinsic and extrinsic motivation. In M. P. Zanna (Ed.), Advances in experimental social psychology (Vol. 29, pp. 271-360). San Diego, CA: Academic Press.

Vansteenkiste, M., Duriez, B., Simons, J., \& Soenens, B. (2006). Materialistic values and well-being among business students: Further evidence for their detrimental effect. Journal of Applied Social Psychology, 36, 2892-2908

Vansteenkiste, M., Lens, W., \& Deci, E. L. (2006). Intrinsic versus extrinsic goal contents in self-determination theory: Another look at the quality of academic motivation. Educational Psychologist, 41, 19-31.

Vansteenkiste, M., Lens, W., Dewitte, S., De Witte, H., \& Deci, E. L. (2004). The "why" and "why not" of job search behavior: Their relation to searching, unemployment experience, and well-being. European Journal of Social Psychology, 34, 345-363.

Vansteenkiste, M., Neyrinck, B., Niemiec, C. P., Soenens, B., De Witte, H., \& Van den Broeck, A. (2007). On the relations among work value orientations, psychological need satisfaction, and job outcomes: A selfdetermination theory approach. Journal of Occupational and Organizational Psychology, 80, 251-277.

Vansteenkiste, M., Ryan, R. M., \& Deci, E. L. (in press). Selfdetermination theory and the explanatory role of psychological needs in human well-being. In L. Bruni, F. Comim, \& M. Pugno (Eds.), Capabilities and happiness. Oxford, England: Oxford University Press.

Vansteenkiste, M., Simons, J., Lens, W., Sheldon, K. M., \& Deci, E. L. (2004). Motivation learning, performance and persistence: The synergistic effects of intrinsic goal contents and autonomy-supportive contexts. Journal of Personality and Social Psychology, 87, 246-260.

Vansteenkiste, M., Simons, J., Lens, W., Soenens, B., \& Matos, L. (2005). Examining the impact of extrinsic versus intrinsic goal framing and internally controlling versus autonomy-supportive communication style on early adolescents' academic achievement. Child Development, 76, $483-501$.

Vansteenkiste, M., Simons, J., Lens, W., Soenens, B., Matos, L., \& Lacante, M. (2004). Less is sometimes more: Goal content matters. Journal of Educational Psychology, 96, 755-764.

Vansteenkiste, M., Simons, J., Soenens, B., \& Lens, W. (2004). How to become a persevering exerciser? Providing a clear, future intrinsic goal in an autonomy supportive way. Journal of Sport \& Exercise Psychology, 26, 232-249.

Vansteenkiste, M., Soenens, B., \& Lens, W. (2007). Intrinsic versus extrinsic goal promotion in exercise and sport: Understanding the differential impacts on performance and persistence. In M. S. Hagger \& N. Chatzisarantis (Eds.), Intrinsic motivation and self-determination in exercise and sport (pp. 167-180). Champaign, IL: Human Kinetics.

Walsh, W., \& Holland, J. L. (1992). A theory of personality types and work environments. In W. B. Walsh, K. H. Cracick, \& R. H. Price (Eds.), Person-environment psychology: Models and perspectives (pp. 35-78). Hillsdale, NJ: Erlbaum.

Williams, G. C., Cox, E. M., Hedberg, V. A., \& Deci, E. L. (2000). Extrinsic life goals and health-risk behaviors in adolescents. Journal of Applied Social Psychology, 30, 1756-1771.

Received May 25, 2006

Revision received March 15, 2007 Accepted March 18, 2007 